\title{
Cosmic-Ray Detection Based on Gray-Scale Morphology of Spectroscopic CCD Images
}

\author{
Jia Zhu ${ }^{\mathrm{A}}$, Zhangqin Zhu ${ }^{\mathrm{A}}$, Chong Wang ${ }^{\mathrm{A}}$, and Zhongfu Ye $\mathrm{A}, \mathrm{B}$ \\ A Institute of Statistical Signal Processing, Department of Electronic Engineering \\ and Information Science, University of Science and Technology of China, \\ Hefei 230027, China PRC \\ B Corresponding author. Email: yezf@ustc.edu.cn
}

Received 2008 November 24, accepted 2008 December 22

\begin{abstract}
Detection of cosmic-ray hits is important because of their disturbing effects on image processing. An algorithm based on gray-scale morphology to detect cosmic-rays in single spectroscopic CCD images is presented in this paper. Based on the morphological differences between cosmic-ray hits and spectra, the operations of erosion and dilation are used to deal with images successively, with appropriate structuring elements. Points of cosmic-ray hits can be detected by analyzing the variation between data pre- and postoperation. Examples of the algorithm are given at the end of this paper, with several groups of both simulated LAMOST images and observed SDSS images to show its performance.
\end{abstract}

Keywords: techniques: image processing — cosmic rays

\section{Introduction}

Astronomical spectroscopic charge-coupled-device (CCD) images are often polluted by cosmic-ray hits during the acquisition process. The disturbing effects of cosmic-ray hits during processing (Offenberg et al. 1999; Axelord et al. 2004; Becker et al. 2004) make it essential to detect and reject cosmic rays from astronomical CCD images.

There are various methods in use to detect and reject cosmic rays. One such direct method is to obtain multiple exposures of the same field to reject cosmic ray hits (Shaw \& Horne 1992). This method was used especially with Hubble Space Telescope data (Windhorst et al. 1994; Freudling 1995). A single pixel will be polluted by a cosmic-ray hit only once or twice in a series of exposures, and by combining multiple exposures we can obtain its replacement value. The disturbance caused by cosmic-ray hits can be eliminated effectively. But the field may be varying, and the positions and intensities of sky lines and object spectra may alter (Croke 1995). Therefore this kind of method is not always available.

Many researchers have concentrated their studies on rejecting cosmic-ray hits in single images, because of the variation in circumstances. One kind of method is based on median-filtering the image (e.g. Dickinson's IRAF tasks QZAP, XZAP). This has recently been improved by Rhoads (2000) by using information about the image's point spread function. Other modified algorithms were proposed later in which neural networks (Salzberg et al. 1995) and interpolation of neighbouring pixels (e.g. the COSMICRAYS task in IRAF) were used. An effective and widely used method based on Laplacian edge detection was proposed by van Dokkum (2001). This approach shows good performance in detection and rejection. Then a fast algorithm based on the histogram of the data was put forward to speed up processing (Pych 2004). Farage \& Pimbblet (2005) evaluated several representative algorithms of the cosmic-ray-hit rejection on single images and gave a high evaluation to van Dokkum's Laplacian algorithm. Zhang et al. (2007) described an algorithm in which an edge-preserving technique called TV in-painting was used to reject the cosmic-ray hits. An effective method based on one-order difference operations and Bessel curve fitting was proposed to detect cosmic-ray hits from single spectroscopic CCD images (Zhu \& Ye 2008).

In this paper, a new gray-scale morphological method is described to detect cosmic-ray hits in single spectroscopic CCD images based on the Large Sky Area Multi-Object Fiber Spectroscopic Telescope (LAMOST) project. Mathematical morphology is an effective tool for image processing and analysis. A detailed demonstration of random sets and integral geometry was given by Matheron (1975), which established the base for mathematical morphology. Serra (1982) perfected the theory of mathematical morphology. Mathematical morphology is a tool for extracting image components which are useful in the description of region shape (Basart, Chacklackal \& Gonzalez 1992) and so it is widely used in many fields, including astronomical image processing. An approach was proposed to solve the automated star/galaxy discrimination problem through the use of mathematical morphology (Candeas, Braga-Neto \& Carvalho Filho 1996, 1997). Then an application of mathematical morphology for the classification of astronomical objects was presented by Moore, Pimbblet \& Drinkwater (2006). Considering the 
morphology of spectra and cosmic-ray hits, using the method of gray-scale morphology to detect cosmic rays is realizable.

We describe the model of spectroscopic CCD image in Section 2, and introduce the principle of gray-scale morphology and details of our method in Section 3. Experiments and results are given in Section 4, in which an application for the observed data of the Sloan Digital Sky Survey (SDSS) using our method is also given. The conclusion is given at the end of the paper.

\section{The Spectroscopic CCD Image Model}

There are various kinds of information included in spectroscopic CCD images, like sky lines, object spectra, system noise, external environment and cosmic-ray hits, and so on. We assume a spectroscopic CCD image model which contains only three kinds of information in the article: spectra including sky lines and object spectra, cosmic-ray hits and noise. Hence, this model is described as follows,

$$
I=S+C+N,
$$

where $S$ represents the spectra, $C$ denotes the cosmic-ray hits and $N$ indicates the noise.

The projection of a spectrum onto the CCD is actually not a point: it expands to its neighbours in the form of a point-spread function (PSF). The PSF of a spectrum is approximately a Gaussian function:

$$
f(x, y)=\frac{1}{2 \pi \sigma^{2}} \exp \left[-\frac{\left(x-x_{0}\right)^{2}+\left(y-y_{0}\right)^{2}}{2 \sigma^{2}}\right],
$$

where

$$
\sigma=\frac{F W H M}{2 \sqrt{\ln 4}},
$$

where $\left(x_{0}, y_{0}\right)$ is the center of the PSF function, $\sigma^{2}$ is the variance and $F W H M$ is short for the full width at half maximum of the Gaussian function.

The PSF of cosmic-ray hits is a non-Gaussian function. The projection of cosmic-ray hits onto the CCD may be a point, or a line, or a spot with a sharp profile. This morphological difference make the detection of cosmic-ray hits in spectroscopic CCD images feasible.

The noise on each pixel point is a Poisson function which does not change the overall morphology of the spectra and cosmic-ray hits. The Poisson distribution parameter is the energy value of the pixel point.

\section{Detection of Cosmic-Ray Hits}

\subsection{Morphological Operation}

Dilation and erosion are the basic operators in mathematical morphology and have become common tools for both image processing and analysis of binary and gray-scale images (Heijmans 1992, 1994, 1995; van Droogenbroeck \& Buckley 2005; Moore et al. 2006; Urbach \& Wilkinson 2008).

We assume that the digital image we are dealing with is $f(x, y)$, and the structuring element is $b(x, y), D_{\mathrm{f}}$ and $D_{\mathrm{b}}$ denote the domains of $f(x, y)$ and $b(x, y)$ respectively.
The gray-scale dilation of the input image $f$ by $b$ is defined as

$$
\begin{aligned}
f \oplus b(s, t)= & \max \{f(s-x, t-y)+b(x, y) \\
& \left.\mid(s-x),(t-y) \in D_{\mathrm{f}} ;(x, y) \in D_{\mathrm{b}}\right\} .
\end{aligned}
$$

This equation is similar to a $2 \mathrm{D}$ convolution, with the 'max' operation and the addition replacing the sums and the products of convolution respectively.

When the image is a simple one-dimensional (1D) function, the dilation operation can be described as follows

$$
\begin{aligned}
f \oplus b(s)= & \max \{f(s-x)+b(x) \\
& \left.\mid(s-x) \in D_{\mathrm{f}} ; x \in D_{\mathrm{b}}\right\} .
\end{aligned}
$$

The value of dilation on each pixel point is the maximum of $(f+b)$ in the neighborhood which is determined by the structuring element $b$.

The dilation operation can be expressed geometrically. The center point of the structuring element slides over the surface of the input image. On each pixel point the output pixel value is the maximum value contained within the structuring element superimposed on the input image (Moore et al. 2006).

The gray-scale erosion of the input image $f$ by $b$ is defined as

$$
\begin{aligned}
f \ominus b(s, t)= & \min \{f(s+x, t+y)-b(x, y) \\
& \left.\mid(s+x),(t+y) \in D_{\mathrm{f}} ;(x, y) \in D_{\mathrm{b}}\right\},
\end{aligned}
$$

which is similar in form to a two-dimensional (2D) correlation, with the 'min' operation and the subtraction replacing the sums and the products of correlation respectively.

For 1D functions, the erosion operation can be simplified as follows:

$$
\begin{aligned}
f \ominus b(s)= & \min \{f(s+x)-b(x) \\
& \left.\mid(s+x) \in D_{\mathrm{f}} ; x \in D_{\mathrm{b}}\right\} .
\end{aligned}
$$

The value of erosion on each pixel point is the minimum value of $(f-b)$ in the interval defined by the sharp of the structuring element $b$.

Erosion and dilation are dual and complementary. To perform the erosion operation, the structuring element slides over the undersurface of the input image. The curved surface formed by the movement of center point is used as the surface of the output image.

The result of performing erosion on a gray-scale image is that the effect of bright details will be reduced when they are smaller in area than the structuring element. And the degree of reduction is determined by the gray-scale values surrounding the bright detail and by the differences of the shape and amplitude values of the detail and the structuring element.

As we have seen, dilation lightens the image and erosion darkens it. We can erode an image by a structuring element and dilate the result by the same structuring element. This operation is called opening which is also an 

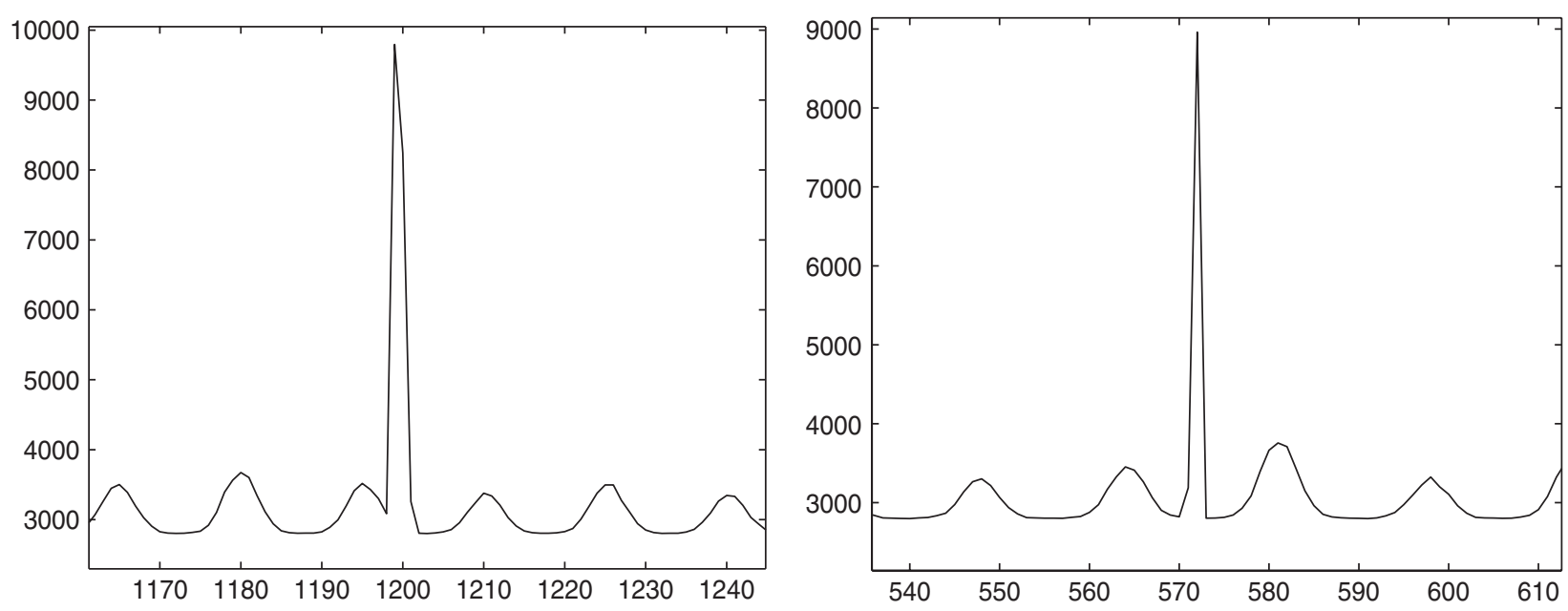

Figure 1 Morphology of spectra with cosmic-ray hits.

important morphological operator. Opening image $f$ by $b$ is defined as

$$
f \circ b(s, t)=(f \ominus b) \oplus b .
$$

The opening operation is idempotent which means that repeated usage of it produces no further effect to the image. Opening is a morphological filter which generally can smooth the contour of the image, break narrow isthmuses, and remove thin protrusions. The degree of smoothing is dependent on the shape and amplitude value of the structuring element.

We assume an image function $f(x, y)$ in threedimensional (3D) perspective, with the third axis being gray-scale values. We also assume that we open the image by a spherical structuring element. Then, we can interpret the principle of opening geometrically as the process of pushing the 'ball' (spherical structuring element) and rolling it against the entire underside of the image surface. The result of opening is that the surface of the highest points reached by any part of the structuring element as it slides over the entire underside of the image surface. The peaks which are narrower than the structuring element in width will be reduced in both amplitude and sharpness. The opening operation can be used to remove small light details in practical applications, while keeping the global gray levels and larger light features almost undisturbed.

The structuring element plays an important role in image morphological analysis. The effect of morphological operations is dependent on the shape of the structuring element. Structuring element can be various shapes including linear, arbitrary 2D flat and 3D shapes in grayscale morphology. The selection of structuring element depends on the shape and size of the object we deal with.

\subsection{Concrete Implementation}

The slices of the spectra including the cosmic-ray hits in spatial direction are shown in Figure 1. We can see that there are some obvious morphological variations between spectra and cosmic-ray hits.

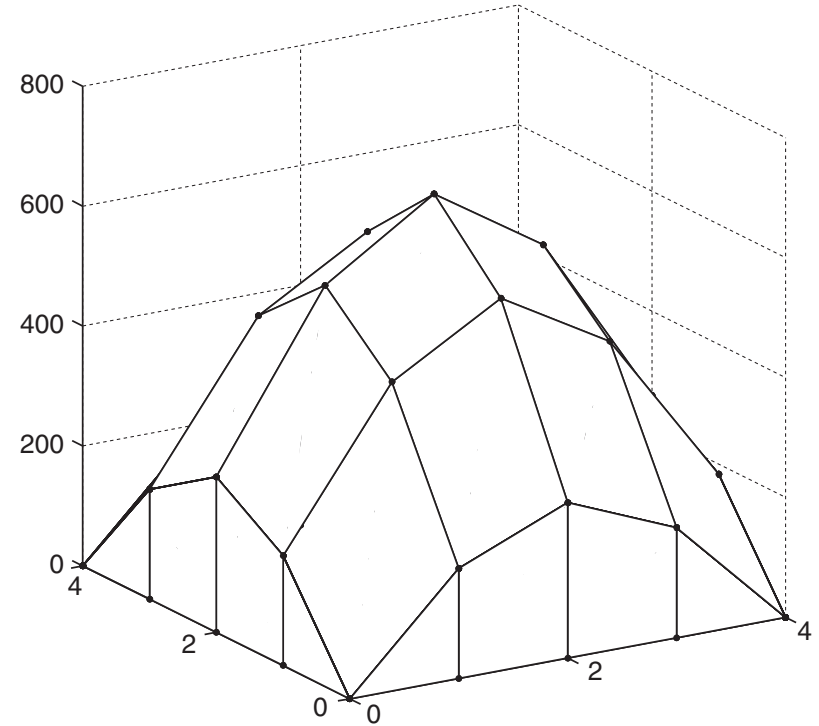

Figure 2 3D structuring element.

The cosmic-ray hits are narrower than the spectral lines in width, even narrower than the $F W H M$ of spectral lines. And the amplitude values of cosmic rays are much greater. Hence, opening this kind of images by a proper structuring element can greatly reduce the cosmic-ray hits in both amplitude and sharpness.

Considering the shape of the spectra, we choose the structuring element based on the PSF function of spectra. The upper surface of the structuring element shown in Figure 2 is approximately a 2D Gaussian function with the same $F W H M$ of the spectra. In the practical operation, the structuring element we choose is a $N \times N$ matrix and the width of the structuring element $N$ is an odd number so that there is a point at the center. The opening operation rejects the small details and keeps larger features almost undisturbed. So that $N$ is not less than the width of the cosmic-ray hits and not more than the $F W H M$ of the spectra, to make sure the rejection of cosmic-ray hits works. When the width of cosmic-ray hits is larger than 

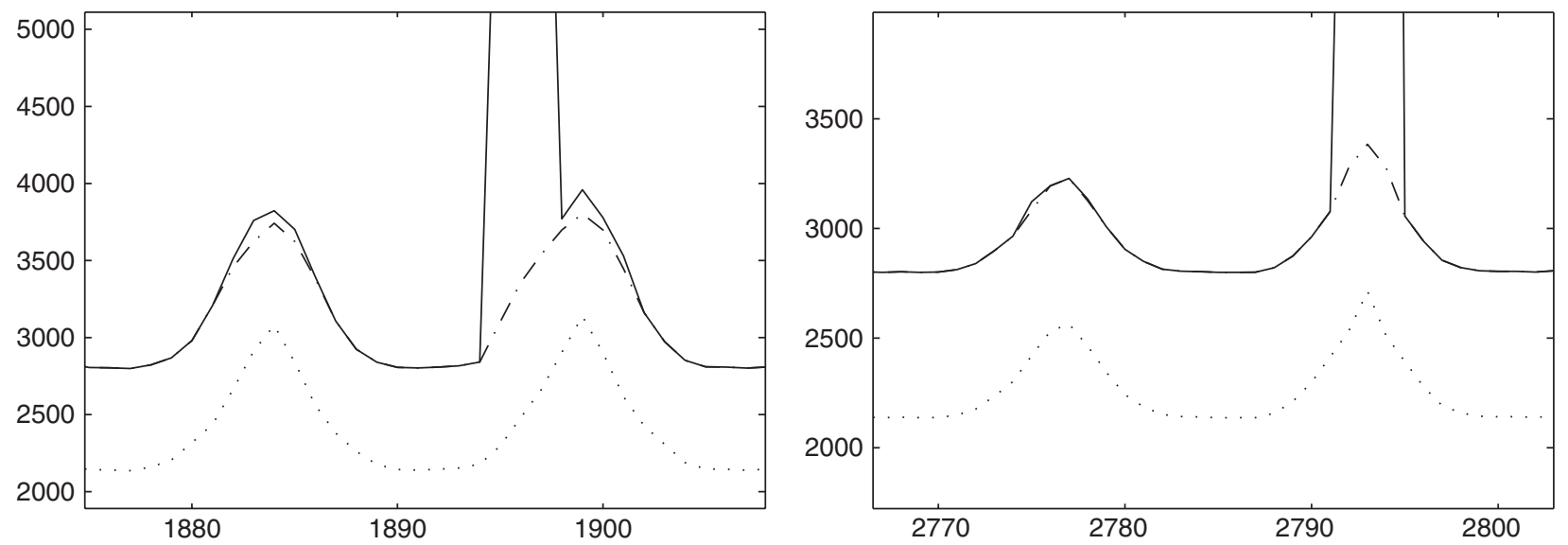

Figure 3 Slices of the results of the erosion and dilation operations. The original image is indicated by a solid line, while the result of the erosion operation is shown by a dotted line. The image after dilating the result of erosion is denoted by a dash-dotted line.
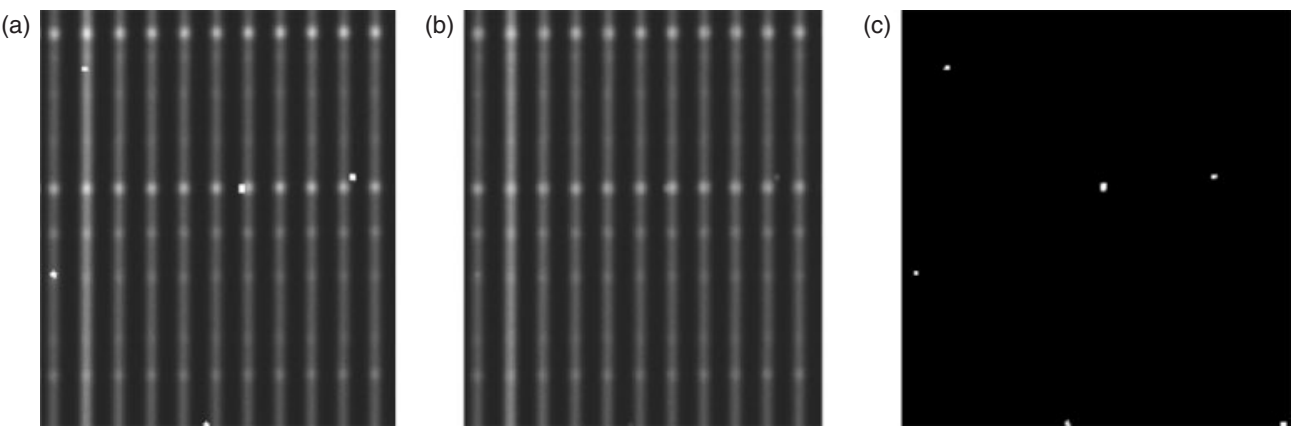

Figure 4 The images pre and post the morphological operation. (a) is the original image with the cosmic-ray hits, (b) is the new image after the morphological operation, and (c) is the difference image of (a) minus (b).

the $F W H M$ of spectral lines, the detection of cosmic-ray hits will be difficult.

The erosion operation will be used to deal with the spectroscopic CCD image first, which can reduce, or even remove cosmic-ray hits with narrow widths and sharp contours. Then we can dilate the result by the same structuring element. This subsequent dilation can increase the brightness of an image which has been darkened by the initial erosion operation, without reintroducing the cosmic-ray hits removed by erosion.

Figure 3 displays the results of erosion and dilation operations for the spectral image with cosmic-ray hits. From Figure 3, we can see that the cosmic-ray details have been rejected after the erosion operation, while the gray value of the spectrum reduced holistically. The dilation operation for the result of erosion almost returns the gray value back to the original level, without reintroducing the cosmic-ray hits.

The images before and after the morphological operation are displayed in Figure 4 to show the effectiveness of the operation intuitively.

Comparing the images before and after the operations, the cosmic-ray hits are removed completely while the spectra are almost unchanged except for a little reduction around the peaks. It is easy to select a threshold based on the differences between the original image and the result, to identify the cosmic-ray hits. Difference value and relative error is the most basic parameter for selecting the threshold. Let the cosmic-ray hits we detect be the confirmation points. At each confirmation point, the pixel value of the original image after the opening operation is used as the value for the output image in which the cosmicray hits are removed. At other pixel points, the output is still the original value before the operation.

Taking into account that a fraction of edge points of the cosmic rays may not be detected easily due to the small residual values, median filtering can be used to improve the detection. After the first morphological operation, the confirmation points are replaced by the median value of their neighborhood. Then we apply the morphological operation to the new image again. With another suitable threshold, these edge points can be detected.

\section{Experiments}

Several simulation data are presented in this section to demonstrate the performance of the algorithm that we propose. A recently proposed method based on Bessel curve fitting (Zhu \& Ye 2008) and the classical algorithm based on Laplacian edge detection (van Dokkum 2001) are both used as the contrast experiments.

The performance differences is measured by the detection probability $P_{\mathrm{d}}$ and false alarm probability $P_{\mathrm{fa}}$ which 
Table 1. Detection efficiency of contrast experiments

\begin{tabular}{lccccc}
\hline Image & \multicolumn{5}{c}{ Group } \\
& 1 & 2 & 3 & 4 & 5 \\
\hline$N_{\mathrm{c}}$ & 8975 & 10689 & 13062 & 8859 & 10912 \\
$P_{\mathrm{d}} \mathrm{L}$ & 0.9207 & 0.8932 & 0.9014 & 0.9586 & 0.9618 \\
$P_{\mathrm{d}}{ }^{\mathrm{B}}$ & 0.9489 & 0.9168 & 0.9248 & 0.9589 & 0.9620 \\
$P_{\mathrm{d}} \mathrm{O}$ & 0.9730 & 0.9502 & 0.9537 & 0.9598 & 0.9693 \\
$P_{\mathrm{fa}}{ }^{\mathrm{L}}$ & 0.0116 & 0.0359 & 0.0610 & 0.0845 & 0.0373 \\
$P_{\mathrm{fa}}{ }^{\mathrm{B}}$ & 0.0011 & 0.0623 & 0.0471 & 0.0001 & 0.0149 \\
$P_{\mathrm{fa}}{ }^{\mathrm{O}}$ & 0.0061 & 0.0512 & 0.0029 & 0.0007 & 0.0228 \\
\hline
\end{tabular}

$N_{\mathrm{c}}$ : quantity of cosmic rays, ${ }^{\mathrm{L}}$ : Laplacian method, ${ }^{\mathrm{B}}$ : Bessel method, o: our method.

are defined as

$$
P_{\mathrm{d}}=\frac{N_{\mathrm{d}}}{N_{\mathrm{c}}} \quad \text { and } \quad P_{\mathrm{fa}}=\frac{N_{\mathrm{fa}}}{N_{\mathrm{c}}},
$$

where the quantity of the cosmic-ray hits is indicated by $N_{\mathrm{c}}$, and $N_{\mathrm{d}}$ denotes the number of correct detection points, and $N_{\mathrm{fa}}$ denotes the amount of false detection points of true objects.

The model of the simulation data with a Gaussian PSF function is based on the LAMOST project. Five groups of simulated images are used here. The simulated images are $4096 \times 4096$ pixels in size, with Poisson noise added to an average background of 2800 counts. The $F W H M$ of the spectra in this five groups of images are different and in a range of 5 to 8 pixels. Group 1 to 3 have more sky lines than Group 4 and 5. The cosmic-ray hits are randomly distributed in the images with the amount of about 10000 pixel points. The orientation angles of cosmic-ray hits are random and the width of each cosmic ray is not more than 5 pixels. The flux levels range from several to 30000 counts in the images. A $5 \times 5$ matrix is used as the structuring element to deal with the images.

The data of contrast experiments are shown in Table 1 .

As we can see from Table 1, the detection probability is improved in comparison to the method based on Bessel curve fitting, while the false alarm probability is still low, even lower. There are more sky lines which often can disturb the detection of cosmic rays in Group 1 to 3 than that in Group 4 and 5. Our method performs more effectively in Group 1 to 3 because it is less sensitive to the sky lines than the method based on Bessel curve fitting. In this contrast experiment, our method shows a better performance in detecting cosmic-ray hits than the method based on Bessel curve fitting. van Dokkum's Laplacian algorithm was proposed for the star/galaxy images. It is very sensitive to the sky lines and bright fiber flux which are ubiquitous in our images.

Some local images are displayed in Figure 5, which can verify the effect of our method intuitively.

In order to prove that our method still performs well in engineering practice, the observed data of SDSS are used. The observed images are $2069 \times 2128$ pixels in size. The
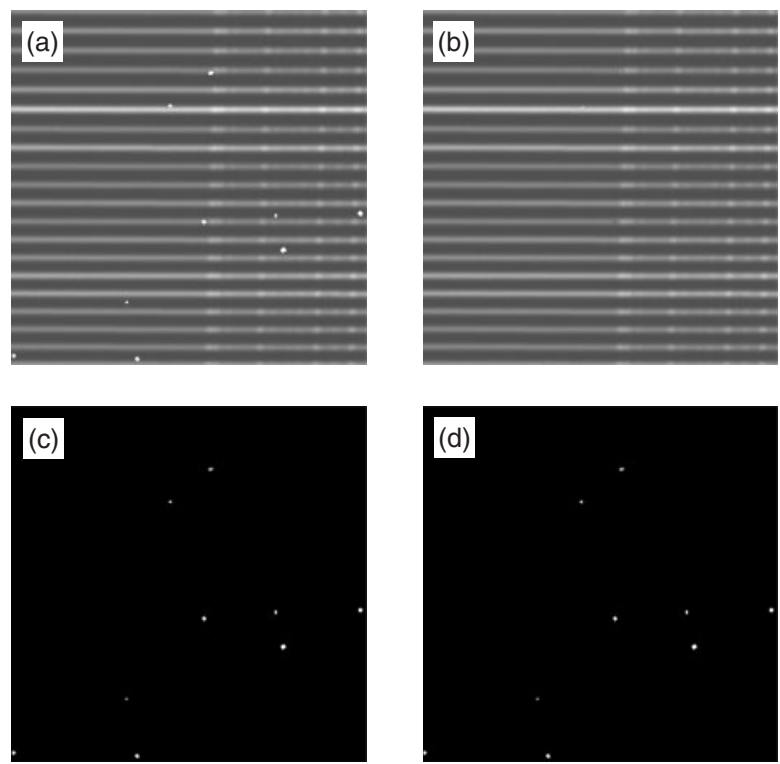

Figure 5 The result of our method for simulation data. (a) is a part of the original spectral image with the cosmic-ray hits, (b) is the same part of the image after our processing, (c) is the cosmic-ray hits detected by our method, and (d) is all the cosmic-ray hits in (a).
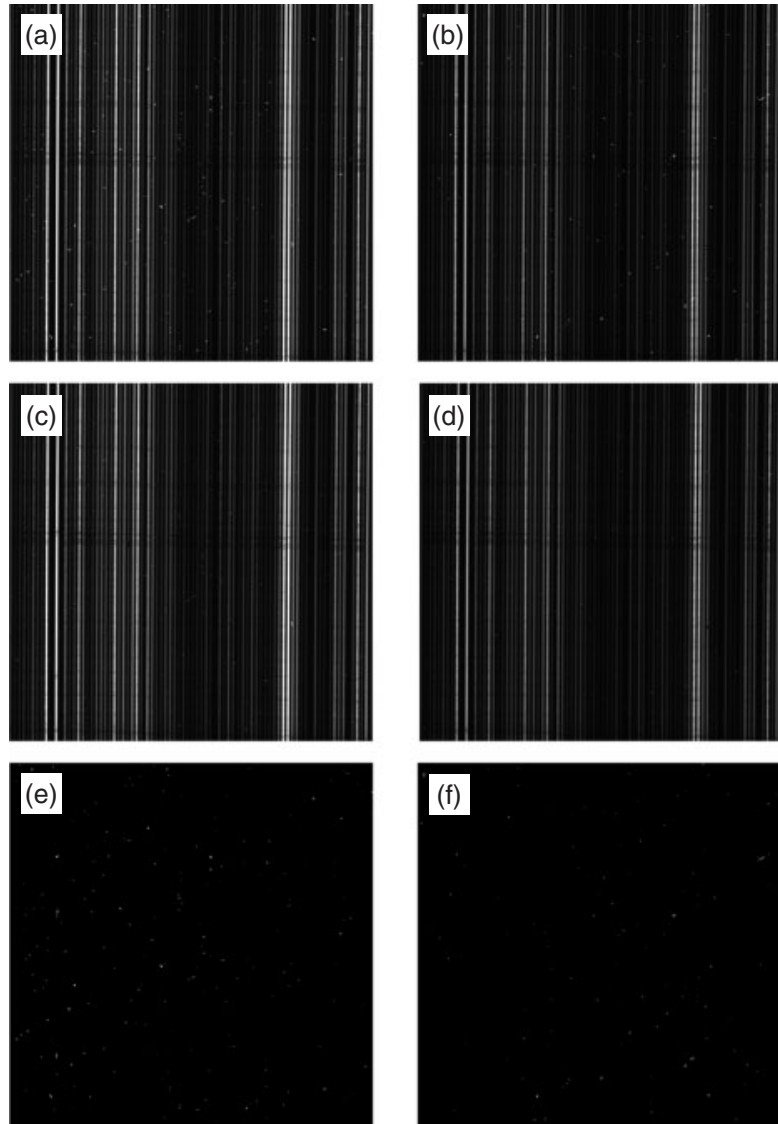

Figure 6 The result of our method for observed data. (a) and (b) are the local images of the original observed SDSS data, and (c) and (d) are the results after processing by our method, and (e) and (f) are the cosmic-ray hits we detected. The two groups of data are observed SDSS data (plate $=2045$, MJD $=53350$ ) sdR-b1-00029795 and sdR-b1-00029796. The domains of local images are both $(600: 1100$, 1500:2000) 
$F W H M$ of the spectra is about 2.8 pixels. We select a $3 \times 3$ matrix to be the structuring element.

The effect drawing of operating the observed data by our method is displayed in Figure 6.

\section{Conclusion}

In this article, a method based on gray-scale morphology has been put forward to detect the cosmic-ray hits from single spectroscopic CCD images. We have introduced a model of the spectroscopic CCD image, described the principles of our morphological operations and analyzed the feasibility of our method. The operations of dilation and erosion can be used to deal with the images, with a suitable structuring element. Cosmic rays can be detected and removed by comparing the data before and after the operation. Experiments given at the end of this paper have demonstrated the effective performance of our algorithm.

\section{References}

Axelrod, T., Connolly, A., Ivezic, Z., Kantor, J., Lupton, R., Plante, R., Stubbs, C., Wittman, D., 2004, in AAS Meeting, 205, 108.11

Basart, J. P., Chacklackal, M. S. \& Gonzalez, R. C., 1992, Advances in Image Analysis, Eds. Mahdavieh, Y. \& Gonzalez, R. C. (Bellingham, Washington: SPIE Press), 306

Becker, A. C., Rest, A., Miknaitis, G., Smith, R. C. \& Stubbs, C., 2004, BAAS, 36, 108.12
Candeas, A. J., Braga-Neto, U. M. \& Carvalho Filho, E. C. B., 1996, Anais do IX SIBGRAPI, 235

Candeas, A. J., Braga-Neto, U. M. \& Carvalho Filho, E. C. B., 1997, J. Brazilian Computer Society, 3, 14

Croke, B. F. W., 1995, PASP, 107, 1255

Farage, C. L. \& Pimbblet, K. A., 2005, PASA, 22, 249

Freudling, W., 1995, PASP, 107, 85

Heijmans, H. J. A. M., 1992, Nieuw Archief voor Wiskunde, Vierde Serie, 10, 237

Heijmans, H. J. A. M., 1994, in Shape in Picture: Mathematical Description of Shape in Grey-level Images, 147

Heijmans, H. J. A. M., 1995, in SIAM Review, 37, 1

Matheron, G., 1975, Random Sets and Integral Geometry (New York: John Wiley and Sons)

Moore, J. A., Pimbblet, K. A. \& Drinkwater, M. J., 2006, PASA, 23,135

Offenberg, J. D., Sengupta, R., Fixsen, D. J., Stockman, P., NietoSantistenban, M., Stallcup, S., Hanisch, R. \& Mather, J. C., 1999, ASPC, 172, 141

Pych, W., 2004, PASP, 116, 148

Rhoads, J. E., 2000, PASP, 112, 703

Salzberg, S., Chandar, R., Ford, H., Murthy, S. K. \& White, R., 1995, PASP, 107, 279

Serra, J., 1982, Image Analysis and Mathematical Morphology (London: Academic Press)

Shaw, R. A. \& Horne, K., 1992, ASPC, 25, 311

Urbach, E. R. \& Wilkinson, M. H. F., 2008, ITIP, 17, 1

van Dokkum, P. G., 2001, PASP, 113, 1420

van Droogenbroeck, M. \& Buckley, M., 2005, JMIV, 22, 121

Windhorst, R. A. \& Franklin, B. E., 1994, PASP, 106, 798

Zhang, L., Bai, Z. R., Wang, C. \& Ye, Z. F., 2007, J. USTC, 37, 688

Zhu, Z. Q. \& Ye, Z. F., 2008, PASP, 120, 814 